\title{
Colloidal epitaxy: Playing with the boundary conditions of colloidal crystallization
}

\author{
Alfons van Blaaderen, ${ }^{* a b}$ Jacob P. Hoogenboom, ${ }^{a b}$ Dirk L. J. Vossen, ${ }^{b}$ \\ Anand Yethiraj, ${ }^{b}$ Astrid van der Horst, ${ }^{b c}$ Koen Visscher $^{c}$ and Marileen Dogterom ${ }^{b}$ \\ ${ }^{a}$ Soft Condensed Matter, Debye Institute, Utrecht University, Princeton Plein 5, \\ 3584 CC, Utrecht, The Netherlands.E-mail: A.vanBlaaderen@phys.uu.nl \\ ${ }^{b}$ F.O.M. Institute for Atomic en Molecular Physics, Kruislaan 407, 1098 SJ, \\ Amsterdam, The Netherlands \\ ${ }^{c}$ University of Arizona, Dept. of Physics, 1118 E. 4th Street, AZ 85721, Tucson, USA
}

Received 29th May 2002, Accepted 7th June 2002

First published as an Advance Article on the web 20th November 2002

\begin{abstract}
We have studied, with quantitative confocal microscopy, epitaxial colloidal crystal growth of particles interacting with an almost hard-sphere (HS) potential in a gravitational field and density matched colloids interacting with a long-range (LR) repulsive potential with a body-centred cubic (BCC) equilibrium crystal phase. We show that in both cases it is possible to grow thick, stacking fault-free metastable crystals: close-packed crystals with any stacking sequence, including hexagonal close packed (HCP), for the HS particles and face-centred cubic (FCC) in the case of the LR colloids. In accordance with recent computer simulations done for HS particles it was found that the optimal lattice constant to grow HS HCP crystals was larger than that of equilibrium FCC crystals. In addition, because of the absence of gravity, pre-freezing could be observed for the particles with the LR potential on a template of charged lines. We also argue that the ability to manipulate colloids with highly focused light, optical traps or tweezers, will become an important tool in both the study of colloidal crystallization and in making new structures. We show how cheap 2D and 3D templates can be made with optical tweezers and demonstrate, in proof of principle experiments with core-shell colloids, how light fields can generate crystal nuclei and other structures in the bulk of concentrated dispersions and how the effect of these structures on the rest of a dispersion can be studied quantitatively in 3D.
\end{abstract}

\section{Introduction}

The crystallization of monodisperse colloidal particles has recently attracted renewed attention because of the increasingly important role colloids play as a condensed matter model system and because the regular 3D structures that can be produced with this form of self-organization find increasing use in the generation of advanced materials such as photonic crystals. The reason for the use of colloids as a model system is that these systems have a well-defined thermodynamic phase behavior that can be manipulated by tuning the inter-particle potential between the colloids through chemical modification and by use of external fields. Moreover, it has recently also become possible to study the structure and dynamics of concentrated dispersions quantitatively in 3D realspace using microscopy techniques such as confocal microscopy. ${ }^{1-4}$ 
In this paper we will focus on the simplest field with which to manipulate colloidal crystallization: the wall of the container in which the crystallization takes place. Already, we have shown in previous work on index matched dispersions of fluorescently labeled core-shell particles that, with a regular corrugation at the wall, the growth of (100) or (110) oriented, stacking fault-free, FCC colloidal crystals of particles with an almost HS potential is possible. ${ }^{2}$ The slow increase in concentration of particles at the wall was the result of sedimentation. Because of the similarities with the growth of molecular crystals on single crystals of another material with a similar lattice constant we termed this method colloidal epitaxy. The gravitational height in the system investigated in ref. 2 was of the same order as the depth of the corrugations at the wall. It is therefore important for an understanding of the mechanism of epitaxial colloidal crystal growth that Heni and Löwen showed by computer simulations and theory that a corrugated surface can induce wetting and pre-freezing of a hard-sphere colloidal crystal at volume fractions that are $30 \%$ lower than those at which the bulk of the liquid freezes. ${ }^{5}$ This is a large effect compared to pre-freezing at a smooth wall which takes place very close $(\sim 1 \%)$ to bulk freezing. Here we will show that particles with (an almost) HS potential in a gravitational field can be grown into any close-packed crystal, including HCP. Moreover, we also investigated the range of lattice spacings at which HCP crystals can be grown and found the optimal spacing to be larger than that of FCC crystals at the bulk freezing transition $(55 \mathrm{vol} \%)$.

Quantitative 3D real-space measurements on systems with LR soft potentials have, as far as we are aware, not yet been done. The reason is that in water it is hard to obtain both index matched systems and Debye screening lengths, $\kappa^{-1}$, that are larger than $300 \mathrm{~nm}$. In this paper we report on a new colloidal model system with which it is possible to obtain $\kappa R$ values $<1$ even for $\mu \mathrm{m}$ size spheres, with $R$ being the sphere radius! The particles we use are the same sterically stabilized PMMA spheres that have featured in many papers before as a model hard-sphere system. The only difference here is that the solvent in which these particles are dispersed is a mixture of cycloheptylbromide and decalin. In this mixture it is possible to match both the refractive index and the density. For brevity, we will only discuss results on LR repulsive potentials where $\kappa R$ is around 2, however, it is possible by addition of salt to turn the potential in this solvent mixture into almost HS-like and in addition dipolar potentials can be realized on top of the LR repulsions with an external electric field. ${ }^{6}$ We therefore feel this system will become an interesting new colloidal model system. Because of the long repulsive range of the interactions we tried a 1D template of charged lines to direct the crystallization. It is probably harder to grow perfect crystals when the templating layer has only 1D order but for possible applications such templates are easier to make. The bulk phase behavior of our LR particle system was BCC and it was found that not only could BCC crystals be aligned with the template, but that the growth of metastable FCC crystals could also be induced.

Although it is more than 40 years since the freezing of purely HS particles was discovered in computer simulations, it is only very recently that in both experiments ${ }^{4}$ and computer simulations ${ }^{7}$ it has become possible to look into crystal nucleation and growth in some more detail and test theories, like 'classical crystal nucleation' theory, that deal with these phenomena. We feel strongly that literally we have only 'scratched the surface' and that progress in the experimental study and manipulation of crystal nucleation, epitaxial growth, defect formation and crystal melting will depend to a large extent on the ability to manipulate colloids with external fields. Although the study in ref. 4 was done without an external field, it is clear that the ability to generate 'unlikely events' or out-of-equilibrium situations experimentally will be instrumental in real-space studies. It is no coincidence that only through the judicious application of 'local fields' have computer simulations in which nucleation barriers can be measured become possible. ${ }^{7}$ Although fields like electric fields at relatively low frequencies (compared to light) and shear fields are also powerful ways to manipulate colloidal crystallization that are explored in our group, ${ }^{8}$ we will limit ourselves here to highly focused light fields with which particles with a higher refractive index than the surrounding liquid can be trapped in $3 \mathrm{D} .{ }^{9}$ Already, we have shown in preliminary experiments that by using acousto-optic deflectors it is possible by time-sharing to keep of the order of $501 \mu \mathrm{m}$ sized particles trapped in a $2 \mathrm{D}$ plane. ${ }^{8}$ In addition, we have demonstrated recently that templates for colloidal epitaxy can be made cheaply just by sticking particles on oppositely charged surfaces with optical tweezers. Even without a high precision $x-y$ table and a control loop with high accuracy particle tracking it is possible to create templates that are accurate enough to direct colloidal crystal 
growth. ${ }^{10}$ Here we want to demonstrate how, by using critical point drying, this method can be extended to 3D structures at a wall. Already the simplest 3D structures are hard to make with more conventional lithographic methods like electron beam lithography, which we used for most of our earlier templates. In proof of principle experiments we will also demonstrate, by using a small quantity of high-index core-shell particles in a concentrated dispersion of index matched particles with a similar outer shell but a fluorescent core, how 2D templates can be generated in the bulk of a dispersion by using optical tweezers and how their effect on the rest of the dispersion can be studied in $3 \mathrm{D}$ with confocal microscopy. Finally, we will show a possible direction to generate structures with optical tweezers in a concentrated dispersion that are not limited to a $2 \mathrm{D}$ plane.

\section{Experimental}

For the HS colloidal epitaxy, templates were made with electron-beam lithography in a $450 \mathrm{~nm}$ thick layer of fluorescently-labeled polymethylmethacrylate (PMMA). ${ }^{2}$ A sample cell, with the template mounted at the bottom, was filled with a low volume-fraction suspension $(\varphi=0.0015)$ of $702 \mathrm{~nm}$ radius silica colloids with a $196 \mathrm{~nm}$ radius fluoresceine-labeled core. After sedimentation, the $3 \mathrm{D}$ structure of the silica particles was analyzed with confocal microscopy. ${ }^{1-3}$ For all experiments on the HS particles, an effective hard-sphere radius, $R_{\mathrm{HS}}$, of $725 \mathrm{~nm}$ was retrieved from the $3 \mathrm{D}$ radial distribution function, $3 \mathrm{D}-g(r)$, determined for a crystal grown on a templateless part of the sample.

For the colloidal epitaxy of the particles with LR potentials, patterns of 1000 lines, $0.550 \mu \mathrm{m}$ wide and $2 \mathrm{~cm}$ long with constant spacing, were made with electron-beam lithography in a $450 \mathrm{~nm}$ thick fluorescently labeled poly(methylmethacrylate) (PMMA) layer. The colloids were fluorescently labeled (dye 4-methylaminoethylmethacrylate-7-nitrobenzene-2-oxa-1,3-diazol (NBDMAEM)), sterically stabilized PMMA spheres of which the synthesis and characterization are described in ref. 11 . In the refractive-index matching solvent mixture used, a $0.74 / 0.26$ volume ratio mixture of cycloheptylbromide and cis-decalin that density matches the spheres to effective $-10^{-2}$ $g$, the sphere diameter is $1.05 \mu \mathrm{m}$. The surface potential was found to be $100 \mathrm{mV}$ with $\kappa R \leqslant 2.5, \kappa$ being the inverse Debye length and $R$ the radius of the spheres. ${ }^{6}$

To probe the phase behavior on the line-template a $100 \mu \mathrm{m}$ thick capillary sample cell, containing a gradient in particle volume fraction of $(4 \pm 1)$ vol\% over $2 \mathrm{~mm}$ after equilibration, was made with the template mounted on the bottom side corresponding to the highest effective gravitational energy.

Both the HS and LR samples were analyzed using fluorescence confocal microscopy (Leica TCSSP2, Leica 100x N.A.). 3D particle coordinates were retrieved as described in ref. 1-3. For specific volume fractions a detailed analysis was performed starting with calculation of the linear number density as a function of distance to the template, $\rho_{z}$, by integration of $3 \mathrm{D}$ particle coordinates over the coordinates parallel to the template. For all volume fractions $\rho_{z}$ showed layering due to the presence of the wall and to crystallization. Layers were defined through the clear minima in $\rho_{z}$. Particles in the same layer were used to calculate $2 \mathrm{D}$ order parameters, such as the $2 \mathrm{D}$ coordinate auto-correlation function (2D-CACF) and its radial average the $2 \mathrm{D}$ radial distribution function $(2 \mathrm{D}-g(r))$. The volume fraction at a specific point in our sample was measured by direct particle counting and checked by measuring the dimensions of the crystal lattice of untemplated crystals.

For the experiments in which 3D structures were made and an optical tweezers array was used in combination with a confocal microscope (Leica TCS NT-2), the tweezers were created by focusing a continuous-wave infrared laser beam (Spectra Physics $10 \mathrm{~W}, 1064 \mathrm{~nm}$ ) to a diffraction-limited spot using a high numerical aperture objective (100x, NA $=1.4$, oil immersion, Leica) on an inverted optical microscope (Leica DM IRB). ${ }^{12}$ The position of the tweezers in the sample was controlled using acousto-optical deflectors (AODs) (IntraAction) or by moving a high-accuracy $(0.5 \mathrm{~nm})$ piezo-electrically controlled microscope stage (Physik Instr.). Apart from the possibility of positioning particles in a single-particle trap, as in the examples shown below, the high scanning speed of the AODs (max. $80 \mathrm{kHz}$ ) also makes it possible to create a time-shared multiple trap, that can trap at least 50 particles simultaneously. The sample cell consists of a bottom glass cover slide and a top substrate with a surface charge opposite to that of the colloids, spaced approximately 10 $\mu \mathrm{m}$ apart. 


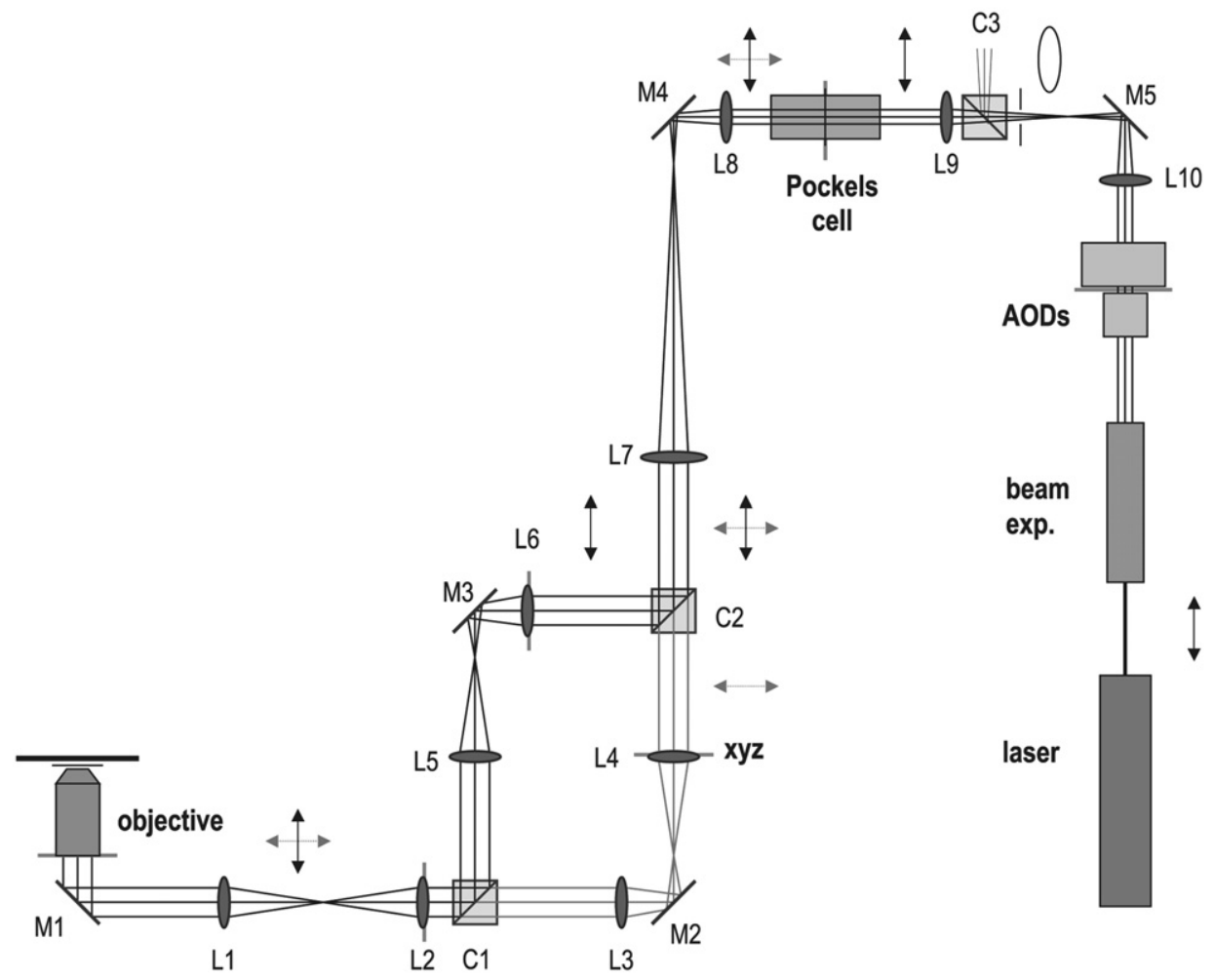

Fig. 1 Schematic diagram of the experimental set-up used to generate, through one lens, two trapping planes at which optical tweezer arrays can be generated by quickly switching a focused laser beam with acousto-optic deflectors (AODs) and a pockels cell.

For the experiments in which colloids were trapped in two planes independently the set-up is given in Fig. 1. Here an infrared laser beam (Spectra Physics 3W CW, $1064 \mathrm{~nm}$ ) is focused in the sample using a high numerical aperture objective (100x, NA $=1.4$, oil immersion, Nikon) creating the optical tweezers. The laser beam is split by a polarizing beam splitter cube. In each of the paths the beam passes through a 1:1 telescope formed by a pair of lenses. The first of the lenses is positioned in a plane conjugate to the back focal plane (BFP) of the objective. Because of this, with every displacement of this lens, which is mounted on an $x y z$-stage, the focus of the beam in the sample moves accordingly, without loss of power. By moving L4 along the direction of the beam, the focus of the horizontally polarized beam in the sample is moved in the $z$-direction with respect to the focus of the vertical polarized beam, and a second trapping plane is created. To have two independent traps, switching between the planes is necessary. For this purpose a Pockels cell (Conoptics, 360-50 LA) is used. The rotation of polarization this electro-optic modulator induces depends on the voltage applied. Alternating the voltage in such a way that the polarization of the laser beam is switched between horizontal and vertical, we can address both planes separately. To create more than one trap in each plane two AODs (InterAction) are inserted in the set-up, again in a plane conjugate to the BFP of the objective. An angle given to the beam here causes an in-plane translation of the focus in the sample. By synchronizing the control of the Pockels cell and the AODs, full control over independent time-shared multiple traps in two planes can be achieved.

To image the two planes using one objective, we moved the camera away from the microscope and inserted a neutral density filter at an angle of $45^{\circ}$. The filter lets through half of the light coming from the microscope, and reflects the other half onto another camera. This second camera is positioned at a longer distance from the microscope in such a way that another plane of the sample is imaged. In this way simultaneous images of the two planes can be obtained. 


\section{Results and discussion}

\section{Colloidal epitaxy of hard spheres in a gravitational field}

The equilibrium crystal structure for hard-sphere-like particles is the close-packed FCC crystal. The free energy difference between this structure and any other possible stacking sequence of close packed planes is however small, of the order of $10^{-4} k_{b} T$ per particle at the melting transition, with the HCP crystal being the most metastable structure. ${ }^{13}$ Due to these small differences, stacking faults are likely to occur and crystallization of hard-sphere-like colloids thus leads to randomly stacked hexagonal close packed (rHCP) crystals. ${ }^{14-16}$

The template used for nucleating the HCP hard-sphere crystal consists of a pattern of holes with the HCP(1100)-symmetry (Fig. 2(a)). This template contains the characteristic $A B A^{\cdots}$ sequence. The lattice vectors are given by $a=2 R \sqrt{ } 3$ and $c=4 R \sqrt{ }(2 / 3)$ with $R$ being the radius of the spheres that would form the corresponding close-packed crystal lattice. The length of the $c$-vector is the typical distance between close packed planes in the HCP-crystal. The coordinates of the $B$ position in the unit cell are $(a / 3, c / 2)$.

In Fig. 3(a) a confocal image of a crystal grown on a template with lattice constants of $a=2.67$ $\mu \mathrm{m}$ and $c=2.51 \mu \mathrm{m}$ is shown. As can be seen the template symmetry, and thus the ABA stacking (Fig. 2(b)) is fully replicated by the silica particles. This shows the growth of a stacking-fault-free HCP crystal. The HCP crystal was found to be monocrystalline over the full area of the template $\left(1.2 \times 1.1 \mathrm{~mm}^{2}\right)$. Furthermore, the HCP crystal structure was found to extend as far as our maximum depth of view inside the crystal, which was approximately $100 \mu \mathrm{m}$. The only defects observed in the crystal were vacancies (indicated in Fig. 3(a)). ${ }^{5}$ In accordance with the simulations without a gravitational field ${ }^{5}$ we found that the optimal lattice spacing to induce HCP crystal growth was
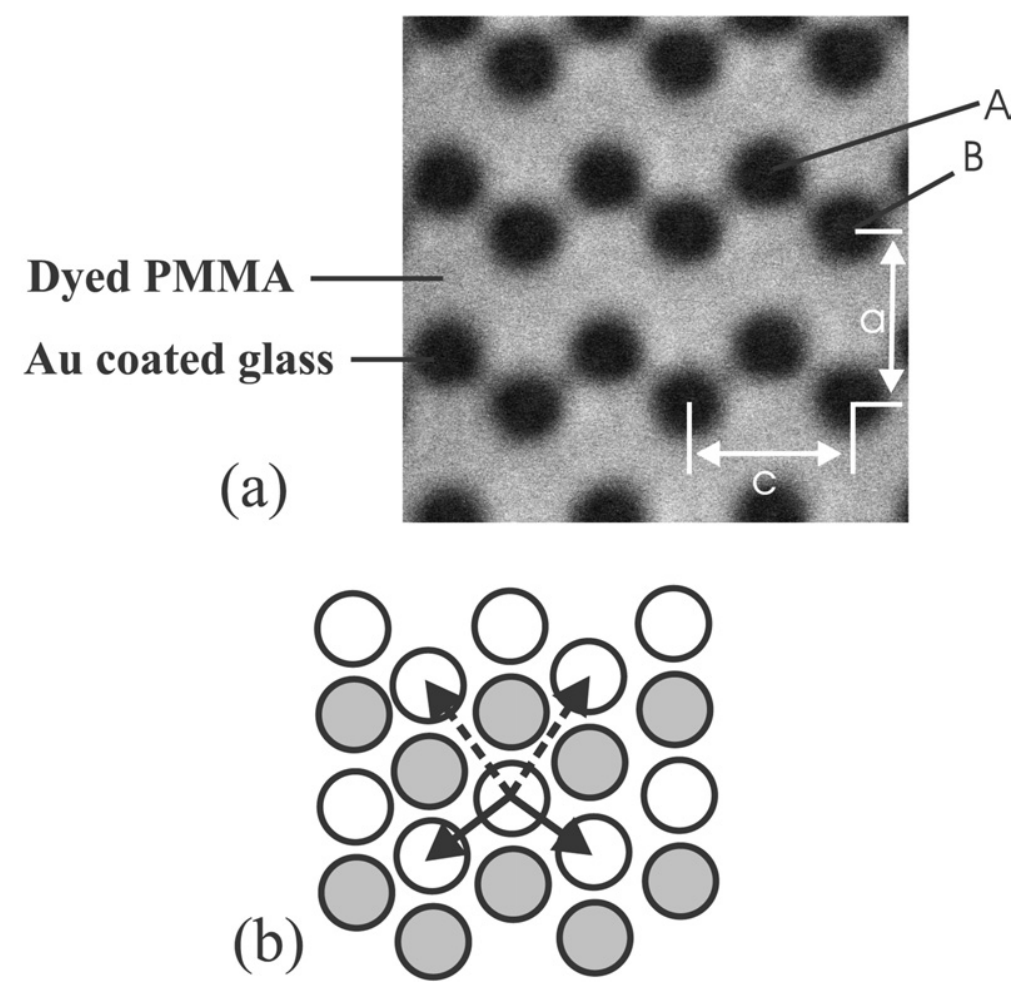

Fig. 2 (a) Confocal microscopy image of an $\mathrm{HCP}(1100)$ template, with the characteristic $\mathrm{AB}$ sequence indicated. The distances $a$ and $c$ span the $2 \mathrm{D}$ unit cell and their lengths are, in this image, $2.08 \mu \mathrm{m}$ and $1.96 \mu \mathrm{m}$ respectively. (b) Drawing of the projection of sphere positions in 2 successive HCP(1100) layers. The solid arrows indicate the in-plane nearest neighbour distance, the dashed arrows the next-nearest neighbor distance. 


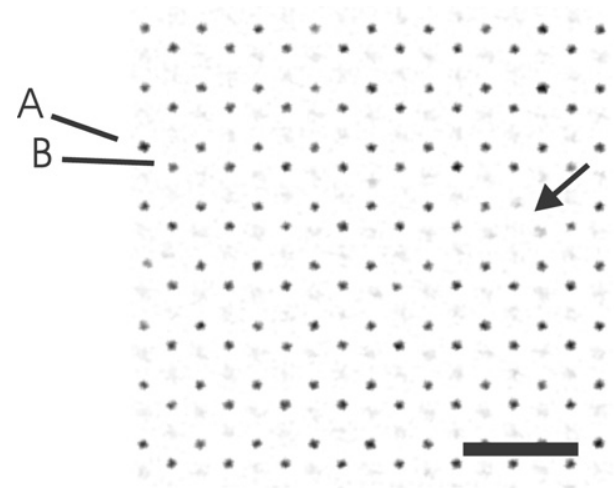

(a)

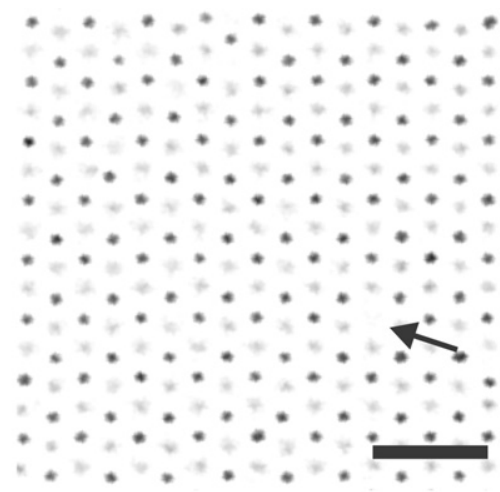

(b)

Fig. 3 Confocal images of (a) the first layer of a templated HCP crystal and (b) the 18th layer in the crystal, with the 19 th layer vaguely visible as well. The arrows indicate the positions of vacancies in the crystal. Scalebars are $5 \mu \mathrm{m}$.

$\sim 5 \%$ larger than the lattice constant at bulk FCC freezing. Apparently, even in a gravitational field the first layer crystallizes significantly below the bulk freezing density and therefore with a larger lattice spacing. We also found that HCP crystal growth could be induced even with lattice spacings that were $\sim 10 \%$ off the optimal value.

As the ABA stacking sequence is dictated by the template and the HCP crystal is the most metastable of all close-packed hard-sphere stacking sequences, this result indicates that it is also possible to grow any other stacking sequence. As an illustration we show in Fig. 4 a so-called double-HCP (dHCP) crystal grown on an $A B C B A$ template.

\section{Colloidal epitaxy of density matched charged spheres with long-range repulsions}

A 1D pattern of lines that has a surface charge similar to that of the colloids was used as a template. The reason for choosing a 1D substrate pattern is two-fold. Firstly, a 1D template is easy to make. Secondly, if perfect epitaxial growth of a $3 \mathrm{D}$ crystal is possible with a $1 \mathrm{D}$ template, we

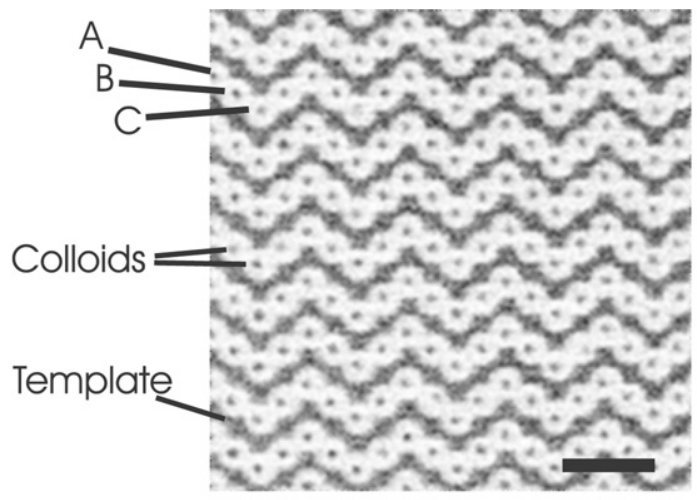

(a)

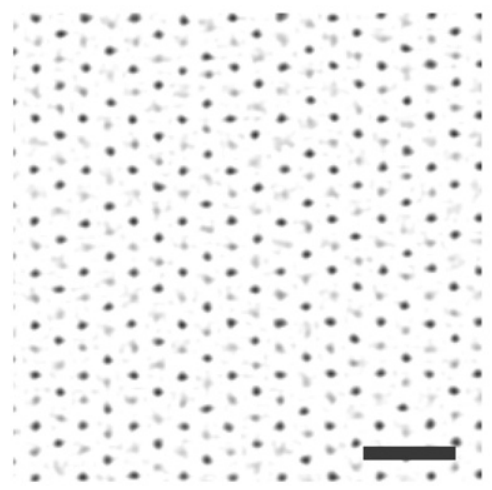

(b)

Fig. 4 Confocal images of (a) template and particles in the first layer of a dHCP (ABCBA) crystal, and (b) the 10 th layer at a depth of $8 \mu \mathrm{m}$. The length of the scalebar is, in both images, $5 \mu \mathrm{m}$. 


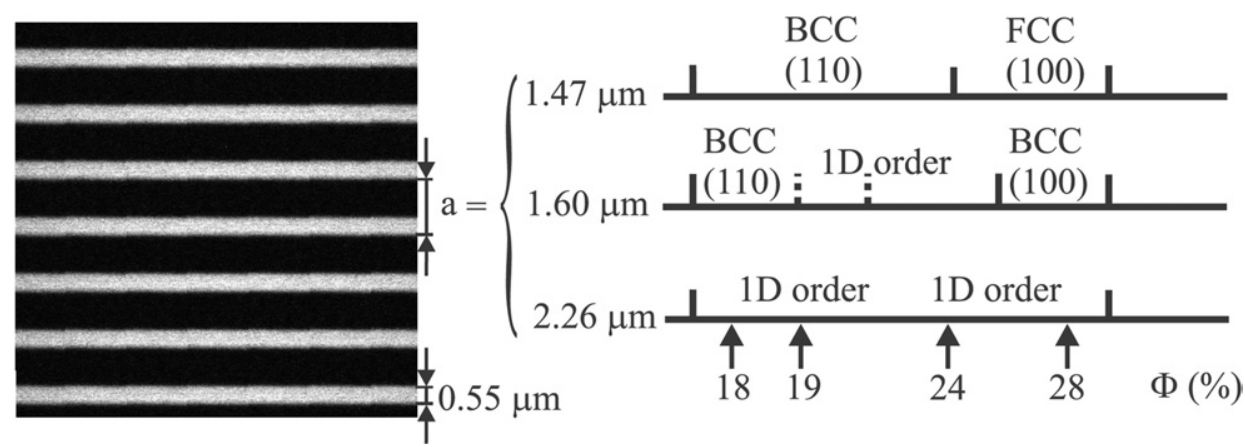

Fig. 5 Confocal image of the striped template pattern (to the left) with the width and spacings of the lines indicated. Light areas correspond to PMMA, dark areas to $450 \mathrm{~nm}$ lower lying ITO-coated glass. For every line spacing the observed surface-layer symmetry is given. For (110)- and (100) planes the 3D crystal structure is also given. Phase boundaries have been indicated with an accuracy of \pm 0.02 , dashed lines indicate a coexistence region between neighboring phases. Volume fractions at which a detailed 3D structure analysis was performed are indicated at the bottom.

would expect a system with LR potentials to be the most successful. A template and the various line spacings investigated are shown in Fig. 5. For all images shown later the orientation of the template is the same as in Fig. 5.

All experiments were carried out at volume fractions where $\mathrm{BCC}$ crystals were found to be the crystal phase that formed at an untemplated wall and in the bulk of the dispersions. The role of gravity in the epitaxial growth process was eliminated by nearly density-matching colloids and solvent and directing the resulting small effective gravitational force (of the order of $-10^{-2} \mathrm{~g}$, with $g$ the earth's gravitational constant) away from the templated wall.

In the following, the structures analyzed with respect to volume fraction and template spacing were classified by the symmetry of the colloids in the surface plane, for which we found three distinct effects. Our first observation is the formation of a square symmetric surface plane that, depending on template spacing, was found to belong to a metastable FCC crystal or a BCC crystal phase. Secondly, we observed alignment of the $\mathrm{BCC}(110)$ plane along the template lines with twinning occurring deeper in the crystal. Finally, the template was found to induce purely $1 \mathrm{D}$ order directed along the lines, but these structures will not be discussed here. The global surface-induced 'phase behavior' is indicated in Fig. 5. At the low volume fraction end of the range investigated $(\varphi \approx 0.10)$ structure was liquid-like, while at the higher volume fraction end $(\varphi \approx 0.30)$, at an untemplated wall, the sample contained BCC crystals only.

Square symmetry (FCC/BCC(100)). $A$ square symmetric arrangement of spheres at the template, as shown in Fig. 6(a), was found at volume fractions between 0.24 and 0.28 and template spacings between $1.47 \mu \mathrm{m}$ and $1.60 \mu \mathrm{m}$. We attribute the local out-of-plane displacements, like in the lower left corner of Fig. 6(a), to stress relaxation due to a small template-crystal mismatch. In the linear number density $\rho_{z}$ (Fig. 6(b)), this effect can be seen as a decrease in peak height and increase in peak width from layer one to four. The structure however remains clearly layered and the interlayer spacing was found to have a constant value of $\Delta z=1.12 \pm 0.01 \mu \mathrm{m}$.

For layers one and four the 2D-g(r) is given in Fig. 6(c). As can be seen, all peak positions are in perfect correspondence with an ideal lattice for both layers. The positions of neighboring particles remain unaltered despite the broadening of layers in $\rho_{z}$. The in-plane nearest-neighbor distance is $d=(1.44 \pm 0.02) \mu \mathrm{m}$, which indeed has a small mismatch with the template spacing of $1.47 \mu \mathrm{m}$. The ratio $d / \Delta z=1.29$ shows that the $3 \mathrm{D}$ structure is a metastable FCC crystal $(d / \Delta z \sim \sqrt{ } 2)$. At similar volume fractions but a larger line spacing of $1.60 \mu \mathrm{m}$, we observed $d / \Delta z$ values of 1.88 , corresponding to a BCC crystal structure $(d / \Delta z \sim 2)$, as indicated in Fig. 5(b).

The average width of the 2D-CACF-peaks (see inset in Fig. 6(a)) in the directions parallel and perpendicular to the template lines was found to be equal within the error margins. Thus combined LR line-sphere interactions for the (100) plane induce 2D crystalline order that bears no 


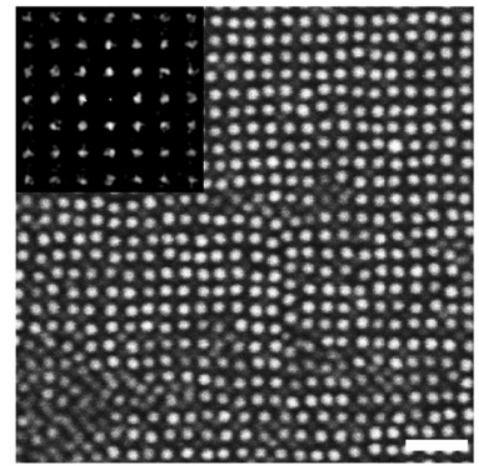

(a)

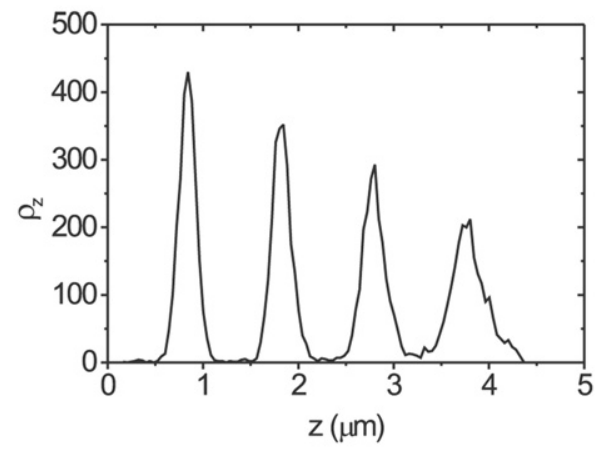

(b)

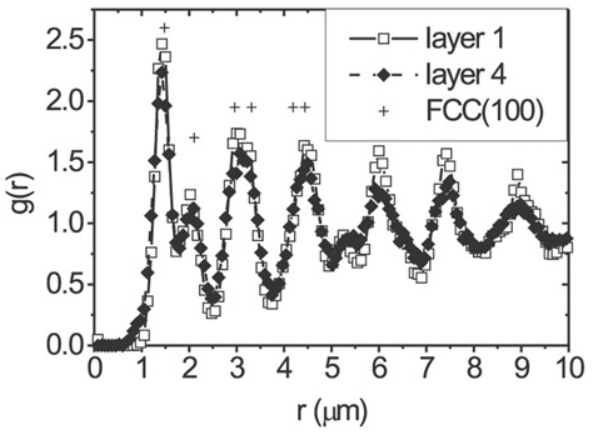

(c)

Fig. 6 (a) Confocal image of the fifth layer in a templated, (100) oriented FCC crystal ( $\varphi=0.28$ and $a=1.47$ $\mu \mathrm{m})$. The inset shows the $2 \mathrm{D}$ coordinate autocorrelation graph of the corresponding surface layer, calculated using the full 3D particle coordinates. Scale bar $5 \mu \mathrm{m}$. (b) The 1D number density in the direction perpendicular to the template. (c) Radial distribution functions for layer one (open squares) and layer four (filled diamonds). Open circles indicate the position of the first six different neighbor-distances in an ideal FCC or BCC (100) plane.

anisotropy of the 1D template anymore. This holds, even though there is a small mismatch between template and interparticle spacings, indicated by the stress relaxation mentioned before. Finally, we want to note that this template was found to induce prefreezing of one to two square symmetric layers at volume fractions where both the bulk structure as well as the surface layers at a plain wall were liquid-like. This is in qualitative agreement with recent findings of Heni and Löwen who showed that, for a hard-sphere system, 2D surface patterns can induce strong prefreezing and even wetting of the crystal. It would be interesting to investigate whether, in the case of hard-spheres, a $1 \mathrm{D}$ template is sufficient to induce prefreezing as well.

BCC(110). At a plain, untemplated wall BCC crystals orient with the densest plane, the (110) plane, parallel to the wall. Fig. 7(a) shows how the line-template orients the BCC(110) plane. Two of the four nearest neighbors, at a distance $d$, are aligned along the direction of the template lines. The other two nearest neighbors can orient at an angle of $+/-54.74^{\circ}$ with the template lines, but the line-template frustrates the position of the two next-nearest neighbors at $1.15 d$ (see Fig. 7(a)). The $3 \mathrm{D}$ orientation of a $\mathrm{BCC}$ single-crystal is given by the striped pattern that is visible when successive BCC(110) layers are being projected (Fig. 7(b)). As can be seen, twinning shows up immediately above the surface layer. The angle between the two orientations was measured to be $110( \pm 1)^{\circ}$, in correspondence with the values given before. 


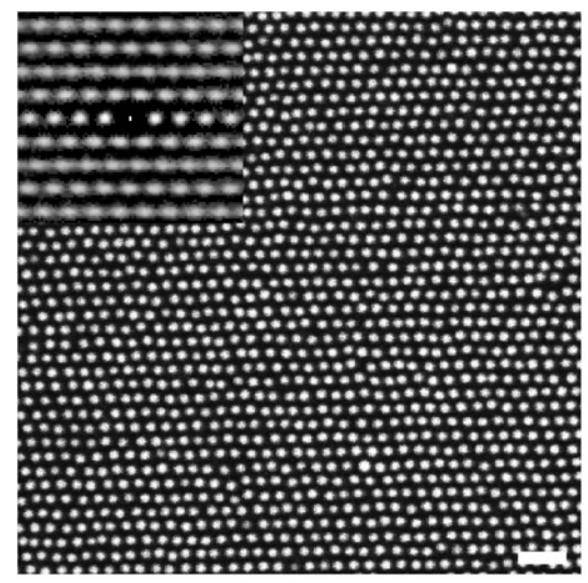

(a)

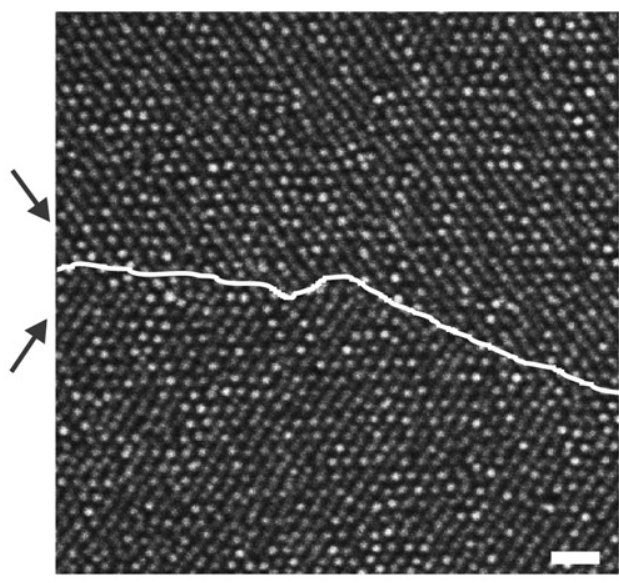

(b)

Fig. 7 Confocal images focused (a) at a surface layer with BCC(110) symmetry, and (b) in-between the second and third layer above (a) $(a=1.60 \mu \mathrm{m}, \phi=0.18)$. The inset in (a) shows the corresponding $2 \mathrm{D}$ coordinate autocorrelation graph. The arrows in (b) denote the two twinned orientations visible in the two-layer BCC(110) projection. The white line indicates the grain boundary. Scale bars $5 \mu \mathrm{m}$.

The 2D-CACF (inset in Fig. 7(a)) shows that positional correlations within the template lines are stronger and of longer range than perpendicular to the lines. The average full-width-at-halfmaximum of correlation peaks up to second order is 1.5 times larger in the direction parallel to the lines than perpendicular to the lines. Contrary to the (100) plane the 1D pattern of lines in this case does not fit with the (110) symmetry, which results in a potential of mean force between spheres in which the 1D template potential can still be recognized.

\section{Making colloidal templates with optical tweezers, on surfaces and in the bulk}

Most of the templates we have used for our studies of colloidal epitaxy were made by electron beam lithography. This is a very accurate, but also slow and expensive, method to make corrugated walls. By using these accurate templates as a mall in a process named soft lithography many templates can be reproduced rather easily and cheaply. However, the use of optical tweezers for the creation of corrugations at a wall is possibly even less expensive and certainly gives more control over aspects, such as materials choice, for the template structure. We have already shown how, by using oppositely charged walls, particles can simply be stuck on the wall in any desired pattern. ${ }^{9}$ An example of a (1100) plane which was used to grow HCP colloidal crystals is shown in ref. 9. There is still a lot of room for improvement with this method and the limits of accuracy and the sizes of the particles that can be placed are not yet known. Of course, these parameters depend strongly on the materials properties, mostly index contrast, of the particles. For instance, we have successfully grabbed $20 \mathrm{~nm}$ diameter silver particles with the tweezers, but this will be much harder with pure dielectric spheres of this size. At the moment we are working on automating the positioning of the particles and using the very high accuracy of piezo positioners $(<1 \mathrm{~nm})$. Such positioners can be used in combination with a feedback loop in which image analysis is used to determine the relative positions of the optical traps, surface features and positioning table. One important extension of the placement of colloids on surfaces is to repeat the procedure and generate $3 \mathrm{D}$ surface features built up from particles. The structures that can be realized by this approach are already impossible, or very difficult, with conventional lithographic methods. An important step in keeping these structures undamaged by the strong forces exerted by the surface tension of the drying liquid in which the structures were made is the use of supercritical drying. In biology this method is used to dry biological samples and in Fig. 8 it is clearly demonstrated that this method also works well to preserve structures made with the optical tweezers. In this figure a pattern with a square symmetry 


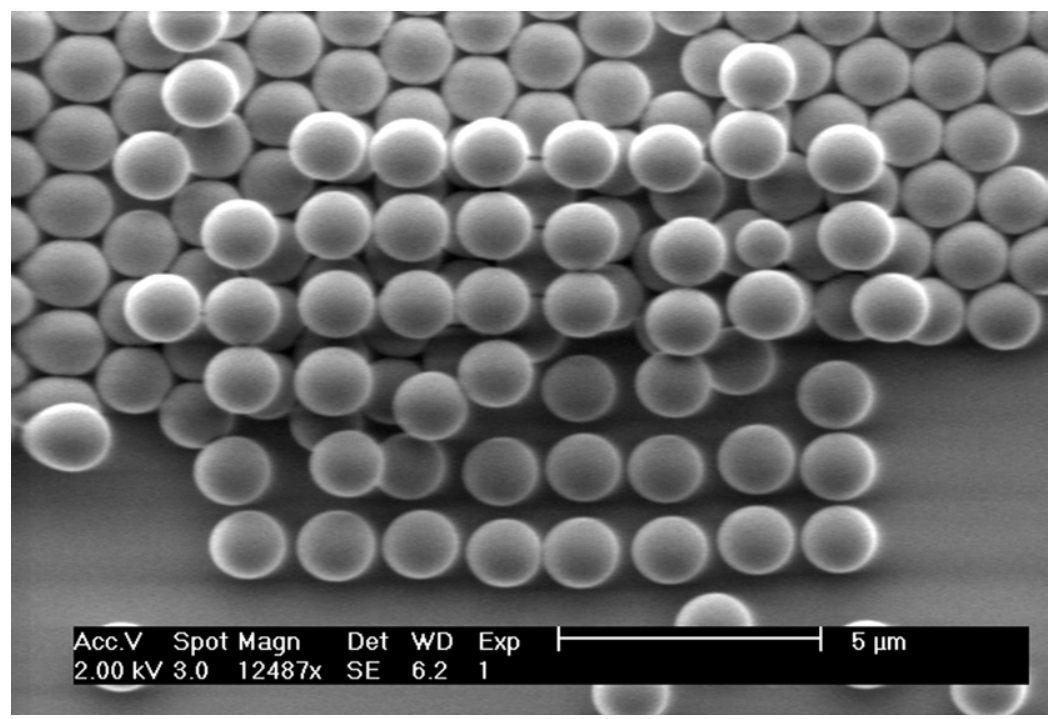

Fig. 8 Square symmetric pattern of $1.4 \mu \mathrm{m}$ diameter silica colloids placed partially over a hexagonal first layer. Structure obtained with optical tweezers by sticking the negatively charged spheres on a positively charged substrate followed by supercritical drying.

was placed with the optical tweezers partly on top of a close packed arrangement of spheres that had formed previously on the glass slide. Without supercritical drying, structures such as those shown in Fig. 8 would have been destroyed by the drying liquid. Now even particles that must be in an almost metastable mechanical arrangement on top of the hexagonal layer stay in the position in which they were stuck by the tweezers. Because particles with different size and/or composition can be placed anywhere with the optical tweezers and more than 100 particles can be positioned at once using time-sharing, this method has much potential. Already it has been shown that, for instance, latex spheres that are sandwiched in-between two silica spheres can be burned away without destroying the remaining stacking of silica spheres.

The use of optical tweezers is much more versatile than just making structures on surfaces. Not only can they be used to measure interaction forces between colloids, they can also be used to selectively form structures in concentrated dispersions of index-matched particles by using tracer particles with a higher index core. A core-shell geometry has important advantages. Not only is it possible to tune the inter-particle interactions with the properties of the shell and for instance make them equal to those of the majority of particles, but the index of refraction of the outer shell can also be matched with that of the surrounding liquid. This index matching of the shell ensures that even if two tracer particles are brought into physical contact by means of the optical tweezers, there are no additional light induced interactions between the colloids. Fig. 9 shows, in green, three particles with a high index $(n \approx 2) \mathrm{ZnS}$ core and silica shell that are held in place with optical tweezers in the presence of a concentrated dispersion of index matched and fluorescently labeled silica spheres that are imaged in red with a fluorescence confocal imaging mode. The high index particles are imaged in confocal reflection mode. The time-averaged frame in Fig. 9(b) shows clearly the influence of the arrangement of the trapped particles on the surrounding non-trapped spheres. With the experimental set-up as described in the Experimental section, the confocal imaging can be performed through an independent lens equipped with a piezo lens scanner, the results of $2 \mathrm{D}$ patterns created by the tweezers can be investigated quantitatively in 3D. We have shown that the use of fast scanning ( $\sim 80 \mathrm{kHz}$ ) acousto-optic deflectors (AODs) allows the placement of $\sim 50 \mu \mathrm{m}$ sized spheres on a $2 \mathrm{D}$ pattern in water without difficulty, by time-sharing the trapping beam. ${ }^{8,12} \mathrm{By}$ using a somewhat higher viscosity liquid these numbers can be significantly enhanced. As in the measurements of interaction forces, the optical tweezers can be used to generate improbable configurations of particles in, for instance a dispersion of particles that is close to the bulk freezing transition. In this way one could study the effects of crystal nuclei with different symmetry. 


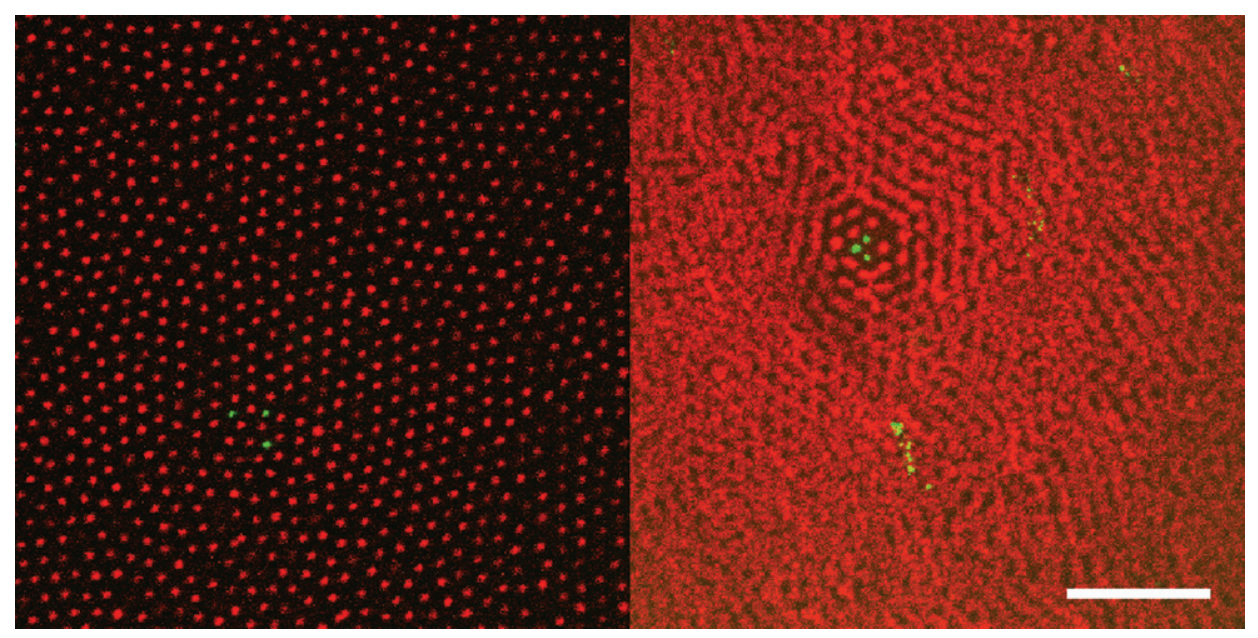

Fig. 9 Combined confocal reflection (green, only tracer particles with $\mathrm{ZnS}$ core visible) and fluorescence (red, only majority particles with fluorescent core visible) microscopy images of a dispersion in which three highindex core-shell particles are kept in place with an optical tweezer array. Image on the left is not averaged and the image on the right is averaged over $16 \mathrm{~s}$ (bar $5 \mu \mathrm{m}$, particle diameter $1.4 \mu \mathrm{m}$ ).

Clearly, one would like to have the ability to generate 3D arrangements of trapped particles as well. One possibility of realizing this is shown in Fig. 1 in which a set-up is described with which two independent $x-y$ time-shared optical tweezer arrays can manipulate particles independently in two different $z$-planes. Because this set-up had not yet been built on a microscope equipped with a confocal scanning unit, the two different planes were imaged by two different CCD cameras. Switching between the different $z$-planes is achieved by using a pockels cell in combination with AODs. In Fig. 10 it is demonstrated that two identical patterns of silica particles trapped in two planes, separated in $z$ by a distance of $\sim 5 \mu \mathrm{m}$ can be moved on top of each other without losing particles in either trapping plane. By combining this approach with the ability to image the surrounding dispersion in 3D one could study the effect of 3D crystal nuclei in a concentrated dispersion. Another approach to achieve the same goal may be the use of programmable holographic arrays as described in ref. 17. Although this method is in principle more versatile, it needs to be seen how many particles can be manipulated by this method in comparison with AODs.

\section{Conclusions}

In this paper we have shown how colloidal crystallization can be influenced by simply changing the boundary conditions, the wall at which crystallization takes place. At a corrugated wall any, metastable, close packed crystal, including HCP, can be grown by choosing the correct $2 \mathrm{D}$ surface pattern for particles with a short-range repulsive potential; for charged particles with long-ranged repulsive potentials $1 \mathrm{D}$ patterns of charged lines can direct the growth of crystals and can also induce metastable crystal growth in this limit of interactions. Thick crystals can be grown epitaxially in the presence and absence of gravity. It will be very interesting to find both experimentally and theoretically to what extent metastable crystals can be stabilized by a templated wall. The strong pre-freezing and wetting found for HS crystallization at a surface with an FCC(111) plane surface corrugation with computer simulations and the pre-freezing of the particles with a LR repulsion at a $1 \mathrm{D}$ template of lines are a strong motivation for further research. One interesting example to explore is the growth of colloidal quasi-crystals.

We have also shown how optical tweezers can be used in several ways in the study and manipulation of colloidal crystallization. First, optical tweezers can be used to generate 2D template structures easily and cheaply. Secondly, 3D surface structures that are much harder to make with conventional lithographic means can also be made by using supercritical drying of the structures made. Finally, in proof of principle experiments we have shown how, by using 


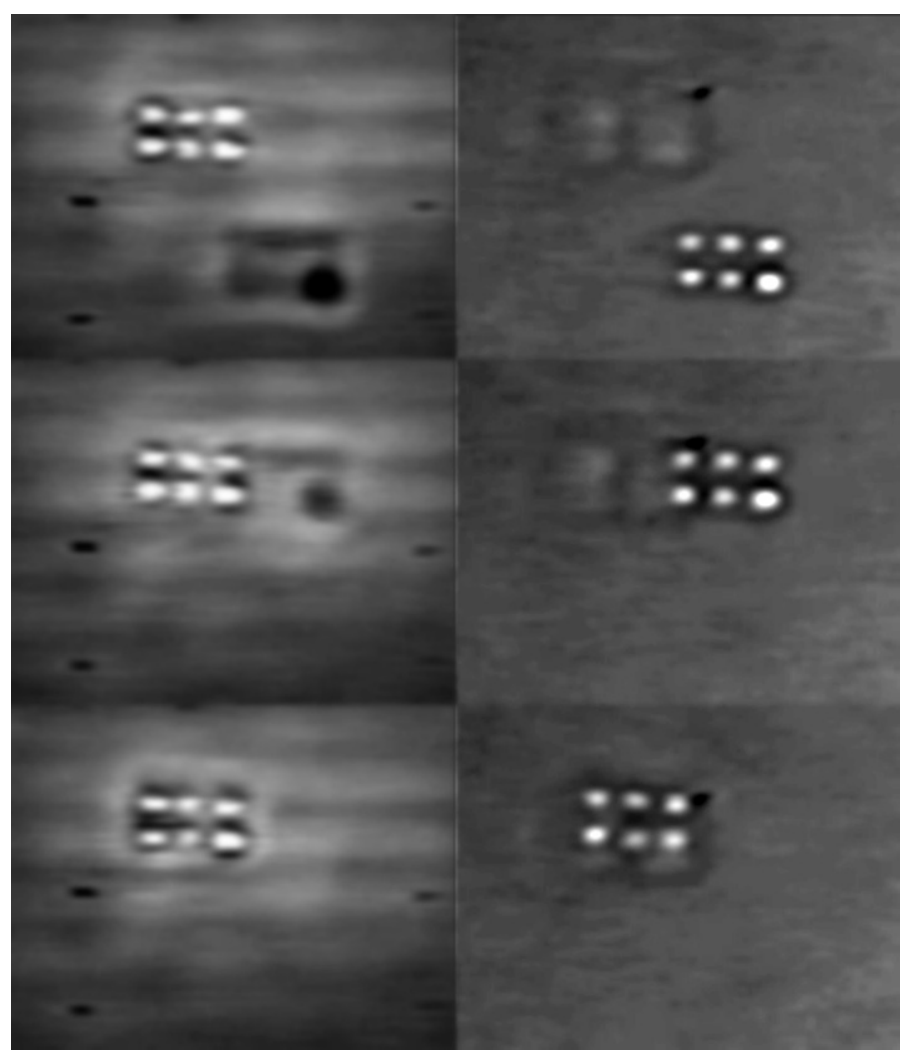

Fig. 10 Video micrographs showing two trapping planes separated by $5 \mu \mathrm{m}$ with 6 silica particles $(840 \mathrm{~nm}$ diameter in water) trapped in the same arrangement $1 \mu \mathrm{m}$ apart in each plane. The left three images are of the lower planes at three different times, the right three images are taken of the upper plane at the same times as those on the left. Vaguely the latex particles in the other planes can be recognized as well. During the three time steps, the patterns are slowly overlapped with their $x-y$ coordinates while no particles are lost from the traps.

dispersions of core-shell particles, the effects in concentrated dispersion of $2 \mathrm{D}$ and even 3D arrangements of particles created by optical tweezers can be studied quantitatively in 3D. These methods will bring experiments on colloidal model systems even closer to computer simulations and will allow for more stringent experimental verification of condensed matter theories.

\section{Acknowledgements}

We are grateful to Anja K. van Langen-Suurling and Hans Romijn (Delft Institute of Microelectronics and Submicron Technology) for their help in creating templates with electron beam lithography and Damir Fific and Karin Overgaag (FOM institute AMOLF) for their help with the creation of some of the structures with optical tweezers. We thank Daan Frenkel for discussions. This work is part of the research program of the "Stichting voor Fundamenteel Onderzoek der Materie (FOM) ", which is financially supported by the "Nederlandse organisatie voor Wetenschappelijk Onderzoek (NWO) ".

\section{References}

1 A. van Blaaderen and P. Wiltzius, Science, 1995, 270, 1177.

2 (a) A. van Blaaderen, R. Ruel and P. Wiltzius, Nature, 1997, 385, 321; (b) A. van Blaaderen and P. Wiltzius, Adv. Mater., 1997, 9, 833.

3 W. K. Kegel and A. van Blaaderen, Science, 2000, 287, 290. 
4 U. Gasser, E. R. Weeks, A. Schofield, P. N. Pusey and D. A. Weitz, Science, 2001, 292, 258.

5 (a) M. Heni and H. Löwen, Phys. Rev. Lett., 2000, 85, 3668; (b) M. Heni and H. Lowen, J. Phys.: Condens. Matter, 2001, 13, 4675.

6 A. Yethiraj and A. van Blaaderen, Nature, 2002, accepted.

7 (a) S. Auer and D. Frenkel, Nature, 2001, 409, 1020; (b) S. Auer and D. Frenkel, Nature, 2001, 413, 711.

8 A. van Blaaderen, K. P. Velikov, J. P. Hoogenboom, D. L. J. Vossen, A. Yethiraj, R. Dullens, T. v. Dillen and A. Polman, in Photonic Crystals and Light Localization in the 21st Century, NATO Advanced Study Institute (Crete, Greece 2000), ed. C. M. Soukoulis, Kluwer Academic, Dordrecht, 2001, p. 239.

9 D. G. Grier, Curr. Opin. Colloid Interface Sci., 1997, 2, 264.

10 J. P. Hoogenboom, D. L. J. Vossen, A. van Blaaderen, C. Faivre-Moskalenko and M. Dogterom, Appl. Phys. Lett., 2002, 80, 4828.

11 G. Bosma, C. Pathmamanoharan, E. H. A. de Hoog, W. K. Kegel, A. van Blaaderen and H. N. W. Lekkerkerker, J. Colloid Interface Sci., 2002, 245, 292.

12 K. Visscher and S. M. Block, in Methods in Enzymology, ed. R. B. Vallee, Academic Press, San Diego, 1997, p. 460.

13 (a) S. C. Mau and D. A. Huse, Phys. Rev. E, 1999, 59, 4396; (b) S. Pronk and D. Frenkel, J. Chem. Phys., 1999, 110, 4589.

14 P. N. Pusey, W. van Megen, P. Bartlett, B. J. Ackerson, J. G. Rarity and S. M. Underwood, Phys. Rev. Lett., 1989, 63, 2753.

15 W. K. Kegel and J. K. G. Dhont, J. Chem. Phys., 2000, 112, 3431.

16 J. X. Zhu, M. Li, R. Rogers, W. Meyer, R. H. Ottewill, W. B. Russell and P. M. Chaikin, Nature, 1997, 387, 883 .

17 E. R. Dufresne and D. G. Grier, Rev. Sci. Instrum., 1998, 69, 1974. 